\title{
GrineColocicical CAncer A seven-step surgical strategy for robotic splenectomy
}

\author{
${ }^{1}$ Division of Gynecology \\ Oncology, Department of \\ Obstetrics/Gynecology, Inova \\ Fairfax Medical Campus, Falls \\ Church, Virginia, USA \\ ${ }^{2}$ Division of Gynecology \\ Oncology, Department of \\ Obstetrics and Gynecology, \\ Walter Reed National Military \\ Medical Center, Bethesda, \\ Maryland, USA \\ ${ }^{3}$ Mid Atlantic Gynecology \\ Oncology and Pelvic Surgery \\ Associates, Fairfax, Virginia, \\ USA
}

Correspondence to Dr Payam Katebi Kashi, Division of Gynecology Oncology, Department of Obstetrics/ Gynecology, Inova Fairfax Medical Campus, Falls Church, VA 22042, USA; pkatebi1@ gmail.com

This video is accepted to be presented at the Society of Gynecology Oncology 2020 annual meeting in Toronto, Canada.

Accepted 10 March 2020 Published Online First 21 March 2020

\section{Check for updates}

(C) IGCS and ESGO 2020. № commercial re-use. See rights and permissions. Published by BMJ.

\section{To cite: Katebi Kashi $P$,} Rojas C, Casablanca Y, et al. Int J Gynecol Cancer 2020;30:1079-1080.

\author{
Payam Katebi Kashi (D) , ${ }^{1}$ Christine Rojas, ${ }^{2}$ Yovanni Casablanca, ${ }^{2}$ Ruchi Garg ${ }^{1,3}$
}

Isolated splenic metastases are rare in gynecologic cancer but may occur due to recurrent uterine or ovarian cancer. ${ }^{1}$ Our objective in this video is to demonstrate the feasibility of robotic-assisted splenectomy for recurrent gynecologic cancer. We examined a case of isolated splenic metastasis successfully excised with robotic-assisted surgery.

Our video demonstrates the seven steps of robotic splenectomy: (1) correct positioning and port placement; (2) splenic flexure mobilization; (3) splenocolic ligament detachment; (4) dissection of the splenic hilum; (5) splenic vessel division; (6) transection of lateral attachments; and (7) removal of the spleen (Video 1). Our patient is a 52 year-old with a history of stage Illa endometrial carcinoma status postrobotic total laparoscopic hysterectomy, bilateral salpingo-oophorectomy, and cytoreductive surgery who presented 16 months after completion of adjuvant chemotherapy with evidence of parenchymal splenic metastasis. Positron emission tomography/ computed tomography was remarkable for a $1.2 \mathrm{~cm}$ splenic lesion concerning for malignancy. She underwent a robotic-assisted splenectomy with operative time of $60 \mathrm{~min}$ and blood loss of $100 \mathrm{~mL}$. Pathology favored recurrent endometrial adenocarcinoma. She was discharged on postoperative day 1 .

Robotic splenectomy for isolated metastasis of gynecologic malignancy is feasible and safe. ${ }^{2-4}$ The enhanced optical quality and dexterity of the robotic system allowed for ideal management with minimal blood loss and improved patient post-operative recovery. ${ }^{2}$
Correction notice Sicne the online publication of this article, the authors have noticed that the twitter handle for Payam Katebi Kashi is missing. This twitter handle has now been added.

Twitter Payam Katebi Kashi @PayamKashiMD

Contributors Performed surgery: PKK, RG. Edited video: PKK, CR, YC, RG. Wrote abstract: CR, PKK. Reviewed the literature: PKK, CR. Final review: PKK, CR, YC, RG. All the authors approved the final version.

Funding The authors have not declared a specific grant for this research from any funding agency in the public, commercial or not-for-profit sectors.

Competing interests None declared.

Patient consent for publication Not required.

Ethics approval IRB for robotic surgery database for the Department of Obstetrics and Gynecology at Inova Fairfax Hospital protocol \#16-2244 submitted and approved.

Provenance and peer review Not commissioned; externally peer reviewed.

Data availability statement Data are available upon request

ORCID iD

Payam Katebi Kashi http://orcid.org/0000-0002-0138-5868

\section{REFERENCES}

1 Telem D, Chin EH, Colon M, et al. Minimally invasive surgery for splenic malignancies. Minerva Chir 2008:63:529-40.

2 Gallotta V, D'Indinosante M, Nero C, et al. Robotic splenectomy for isolated splenic recurrence of endometrial adenocarcinoma. J Minim Invasive Gynecol 2018;25:774-5.

3 Gallotta V, Nero C, Lodoli C, et al. Laparoscopic splenectomy for secondary cytoreduction in ovarian cancer patients with localized spleen recurrence: feasibility and technique. J Minim Invasive Gynecol 2016;23:425-8.

4 Giulianotti PC, Buchs NC, Addeo P, et al. Robotassisted partial and total splenectomy. Int $J$ Med Robot 2011;7:482-8. 
Video article

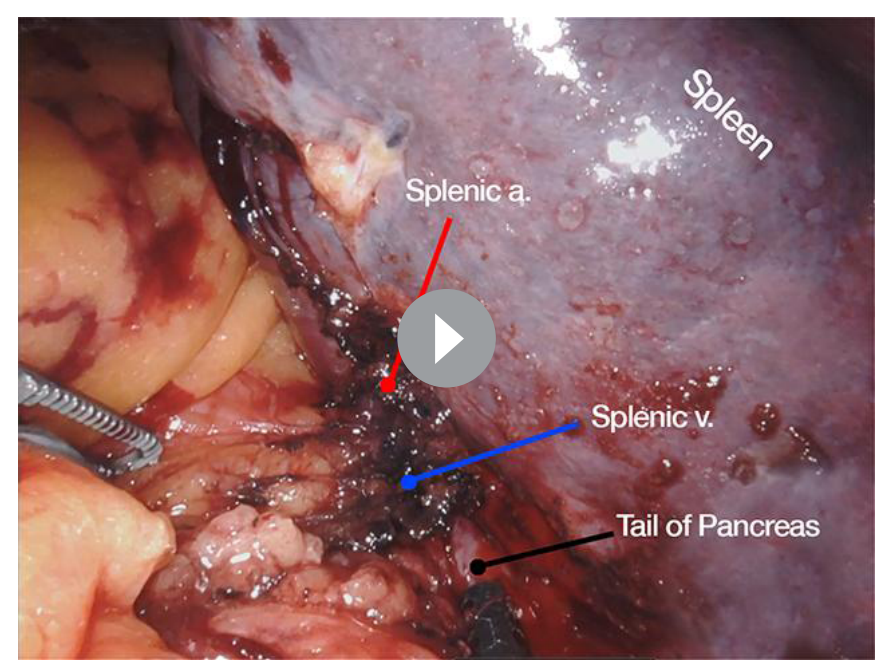

Video 1 Robotic-assisted splenectomy for recurrent gynecologic cancer. 